\title{
Presidential address Knowing and doing in the sixteenth century: what were instruments for?
}

\author{
JIM BENNETT*
}

\begin{abstract}
Despite recent work on scientific instruments by historians of science, the meeting ground between historians and curators of collections has been disappointingly narrow. This study offers, first, a characterization of sixteenth-century mathematical instruments, drawing on the work of curators, as represented by the online database Epact. An examination of the relationship between these instruments and the natural world suggests that the 'theoric', familiar from studies of the history of astronomy, has a wider relevance to the domain of practical mathematics. This outcome from a study of collections is then used in re-examining an established question in the history of science, the position of William Gilbert on the motion of the Earth.
\end{abstract}

It is an irony of the current vogue for instrument studies in the history of science that there has been little impact on those who care for instrument collections. Custodians of the accumulated scientific hardware long complained that historians were overly focused on ideas and neglected the material culture that shaped so much of scientific practice. Now that such a complaint is no longer tenable, do they feel vindicated and appreciated? No. Instead they report that historians still make little use of surviving instruments as resources for research. Their public continues to be overwhelmingly the general visitors to museums and the specialist collectors or instrument enthusiasts, while they are underwhelmed by the demands of historians for research access to collections.

At the same time, it is difficult not to feel that curators should be seizing the opportunities presented by the instrumental turn in historical writing, and engaging more noticeably with the new historical agenda. Curators have not been prominent in their contributions to this development. To be fair, their professional lives and the demands on their time have changed profoundly as many museums have moved away from traditional expertise, scholarship and intellectual endeavour. An ambition to be inclusive and populist, which museum staff will generally share as they seek to offer their visitors a rewarding experience in their galleries, can result in the agenda being overtaken by interests from outside collections - from politics, commerce and science - and

* Museum of the History of Science, University of Oxford, Oxford, OX1 3AZ, UK.

This address was delivered at the BSHS meeting 'Do collections matter to instrument studies ?' at the Museum of the History of Science, June 2002. 
galleries becoming tools of social policy, of opinion formation, or even of advertising. At a time when historians have become more interested in instruments, museum authorities have been removing collections from galleries to make room for displays of a type that can exert more control over the visitor's experience.

Although the apparent promise of more interaction between historians and curators has not been realized, this address is an opportunity to propose the sixteenth century as a promising occasion for dialogue. Significant developments for historical and curatorial work can be located in a period where an accurate characterization of the changing role of instruments requires the work of both expert communities. Historians have been much more attracted by the instrumentation of the seventeenth century - by air-pumps, telescopes, microscopes, barometers and so on. For one thing, historians are right to feel that in 1600 or thereabouts they are able to begin with a clean sheet, since there are new departures in optical instruments and in instruments of natural philosophy. Further, these are entangled with intellectual debates about cosmology and experimental philosophy, carried on in the literary culture of the time and reconstructed through the written record - the books, the emerging journals, the records of the new learned societies, the correspondence and other literary remains of the practitioners. It is perhaps to the advantage of the historical mode of work, and to the disadvantage of the curatorial, that very little survives of all this instrumentation. There are plenty of seventeenth-century instruments, certainly, but not many of them belong in optics and natural philosophy. The great majority fall into the traditional class of mathematical instruments, where the narrative is very far from beginning on a clean sheet, where there is a long history before 1600 that, despite the efforts of curators in caring for their collections, is still not taken on board or properly understood in the history of science.

While maintaining that the material record of the new types of instrument in the seventeenth century is slight, we can admit that in the case of optical instruments there are a couple of Galileo telescopes, a few telescopes by Johann Wiesel, components from Christiaan Huygens, a fragment from Newton, some microscopes from Antoni van Leeuwenhoek and some telescopes and microscopes by commercial makers such as Giuseppe Campani, John Yarwell, John Marshall and so on. But overall this does not amount to very much, especially not in its original state. There is very much less if we search for the material remains of the experimental philosophy, although the survival of the delicate thermometers from the Accademia del Cimento in Florence is extraordinary. ${ }^{1}$ While optical instruments found a public in the seventeenth century, instruments of natural philosophy did not become common items of commerce until the eighteenth.

In the sixteenth century one might say that the historical and curatorial programmes can be more evenly balanced. They can box, so to speak, in the same weight division. There we might find a more auspicious testing ground for the question of whether collections can be important to instrument studies. But it is not only to find a more level playing field that we might look at the sixteenth century; it is as much because there are

1 M. Miniati (ed.), Museo di Storia della Scienza: Catalogo, Florence, 1991, 132-47. For seventeenthcentury instruments in general, see M. Daumas, Scientific Instruments of the Seventeenth and Eighteenth Centuries and their Makers (tr. M. Holbrook), London, 1972. 
lessons to be learned from the instruments of the period that have a general and illuminating significance for the changes characteristic of the Scientific Revolution.

Thanks to the work of the curators we have a fair acquaintance with the burgeoning range of mathematical instruments of the sixteenth century. Adding the qualifier 'mathematical' does not restrict the class of instruments under consideration - in the terminology of the period, there are only mathematical instruments - but using it is a valuable reminder that these are not 'scientific instruments' in our broader sense. Neither, of course, are they 'mathematical instruments' in our narrower sense; that is, they are not just for drawing and calculation. They are for astronomy, surveying, navigation, warfare, architecture, gnomonics and so on, as well as for drawing and calculation. Curators now have a fair knowledge of them - of the range of designs and how they work, the centres of production, the individual makers and so on. While this familiarity has not extended to the historians, the curatorial side, for all its technical knowledge, still lacks a sympathetic and sufficiently subtle and unprejudiced characterization of what these instruments were meant to be: we still take too much for granted about their nature and purpose.

This address first offers some thoughts about the characterization of the mathematical instruments of the sixteenth century. These will be illustrated through one of the results of recent curatorial work, an online catalogue of instruments from four museums offered under the title of Epact. ${ }^{2}$ These curatorial observations will then provide the basis for some fresh thoughts on an old problem for historians of science concerning the cosmology of William Gilbert, the overall aim being a fruitful combination of the curatorial and historical modes of work.

The distinction between knowing and doing can be a first step towards finding an appropriate characterization for the mathematical instruments. Today we think of many types of scientific instrument in terms of knowing, by giving them roles such as discovery or detection, but this was not the case in the sixteenth century. Insights into the natural world, its unseen structures and organizing principles, its causal links, its formal and material constitution, belonged with natural philosophy, whereas instruments belonged with mathematics. It is immediately evident from even a superficial study of the surviving instruments that they are for doing: they offer their services to a range of activities, often those populated by professional practitioners, such as surveyors, navigators, astrologers, military engineers and so on, but often also for more everyday needs such as telling the time. What the instruments do, or what people do with them, is find answers to certain problems. They do not discover things, except in so far as it can be said that the time or the latitude is 'discovered' by being measured. In the more fundamental and indeed literal sense of 'dis-cover', users of instruments do not increase their knowledge of the natural world in the sense characteristic of natural philosophy.

One very telling instance of this is that pre-Copernican terrestrial globes rotate on polar axes - not because their makers and users were proto-Copernicans and thought that the Earth itself behaved like that, but because it was very convenient for getting the

2 Available via the internet at www.mhs.ox.ac.uk/epact. 


\section{Jim Bennett}

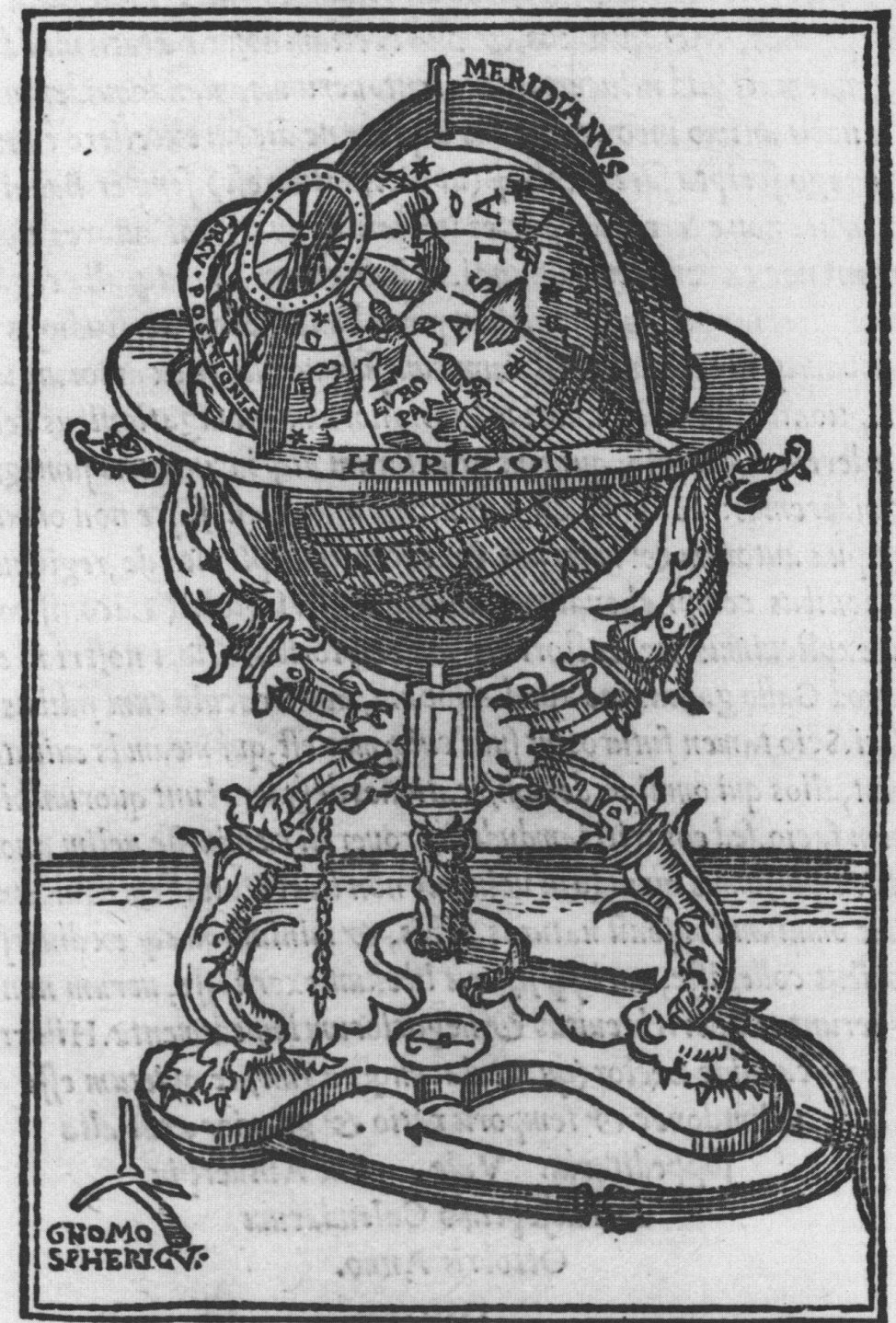

Figure 1. Gemma Frisius's rotating terrestrial globe from his De principiis astronomiae \& cosmographiae of 1530: 'we can do by geometrical invention what is not permitted in the natural world' (see note 9).

kind of answers that globes could deliver if they rotated (Figure 1). ${ }^{3}$ Of course, celestial globes from the same period did the same.

3 For an early illustration of such a globe, see Gemma Frisius, De principiis astronomiae \& cosmographiae, Antwerp, 1530. 


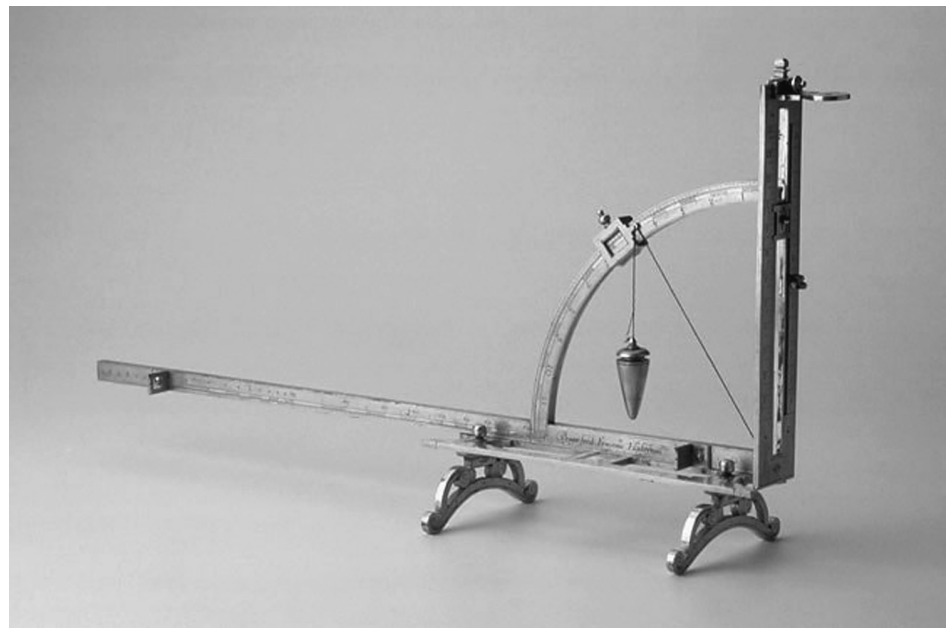

Figure 2. Gunnery instrument incorporating an artillery level and sight by Erasmus Habermel, late sixteenth century. Museum of the History of Science, inventory no. 41591.

We can say then that these instruments are practical, but only in the sense that they purport to be for solving problems. Because the second thing that is evident from their study as surviving objects is that in a good many cases they appear not to be very practical at all. Some seem to be as much for making an impression as for solving any immediate problem. They do not confine themselves to what is practical but extend far into what is ingenious and what is decorative. Epact has a great many instruments in this class, partly because, since they are valued and protected, they survive in disproportionate numbers. They may be elaborate, richly decorated, unnecessarily complex or impossibly delicate for the life of robust action they profess to assist (Figure 2).

In fact these instruments are part of a complex mathematical economy with many layers and interconnections, so it is not surprising that they resist simple, one-dimensional characterization. Makers are providing products for different types of client and patron in very different circumstances, while the makers themselves have very different aspirations. There is no sharp division between makers and mathematicians - some of the most able and prestigious among them, such as Johannes Regiomontanus, or Gerard Mercator, or Egnatio Danti or Gemma Frisius, were happy to make instruments, to present themselves as makers, or to preside over workshops and printing presses. In the case of one instrument in the Epact database that has particularly interested me, the maker was a member of the aristocratic Pitti family in Florence and Abbot of the monastery of San Miniato al Monte overlooking the city. ${ }^{4}$

Despite the scope for complexity and sophistication - even for exaggerated sophistication-there is a sense in which mathematical instruments are confined to the practical in that they are not about natural knowledge. It might be said that there is knowledge here - knowledge of geometry, of making and using, of the arrangement of

4 J. Bennett, 'Epact unpacked: the sundials of Miniato Pitti’, Sphaera (1998), no. 8, 2-3. 


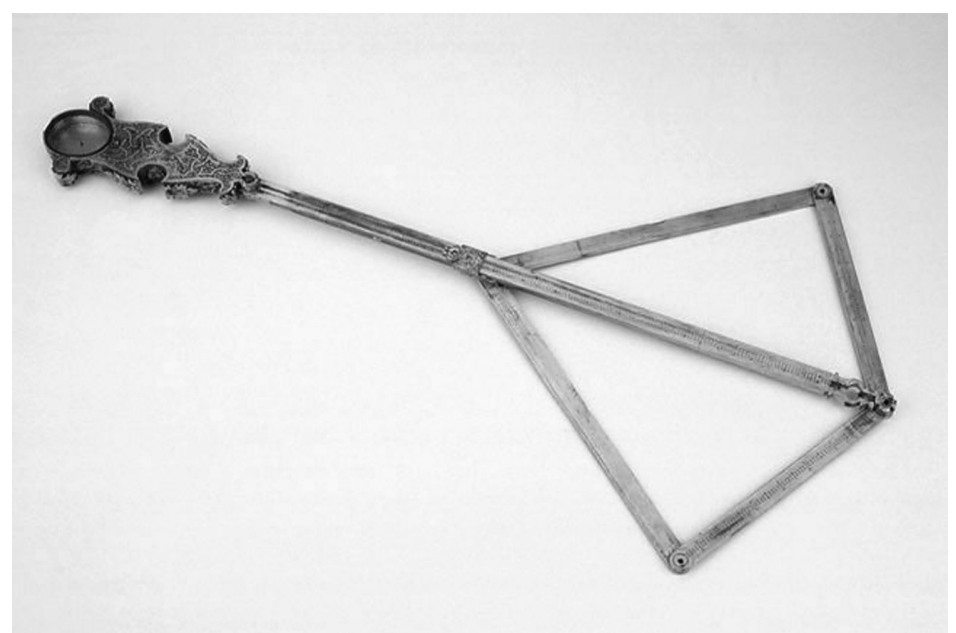

Figure 3. The radio latino, a multi-purpose instrument for warfare, by Giovanni Maria Mancini, c. 1600. Museum of the History of Science, inventory no. 37525.

the stars and the distribution of the world. But it is important to be careful about the knowledge claims being made. We might consider, for example, the central discipline of cosmography, and its distinction from cosmology. Cosmography combined astronomy, geography, surveying, navigation, cartography and instrumentation and concerned itself with the representation of both the heavens and the Earth, but unlike cosmology it did not deal with the natural philosophy of either. It fell within the domain of practical mathematics and the people concerned with it were typically those interested in instrumentation and cartography, such as Sebastian Münster, Gerard Mercator, Peter Apian or Gemma Frisius.

In characterizing practical mathematics between knowing and doing, then, we must first say that in so far as instruments do deal with knowledge, it is not knowledge of natural philosophy, it is not knowledge of the material and causal structure of nature. Further, at a rhetorical level, practical mathematicians often emphasize doing rather than knowing in any sense. There are lots of examples from the Epact database, but a dramatic one is the 'radio latino', an instrument for the military surveyor and the gunner, used for setting artillery or for laying out a fortification (Figure 3). Part of its material rhetoric is that it is designed to be kept in a scabbard and drawn like a sword (Figure 4). ${ }^{5}$ There are even instruments that could actually be used as weapons. ${ }^{6}$ Another instance would be the triangulation instruments, such as those of Joost Bürgi, Erasmus Habermel (Figure 5) or Leonhard Zubler: when they are illustrated in contemporary textbooks or manuals they are often shown range-finding in the immediacy of a conflict. ${ }^{7}$

5 E. Danti and L. Orsini, Trattato del Radio Latino, Rome, 1583.

6 Miniati, op. cit. (1), 16; B. Romano, Proteo Militare, Naples, 1595.

7 See, for example, L. Zubler, Novium instrumentum geometricum, Basel, 1607; B. Bramer, Bericht zu Jobsten Burgi seligen geometrischen triangular Instruments, Kassel, 1648. 


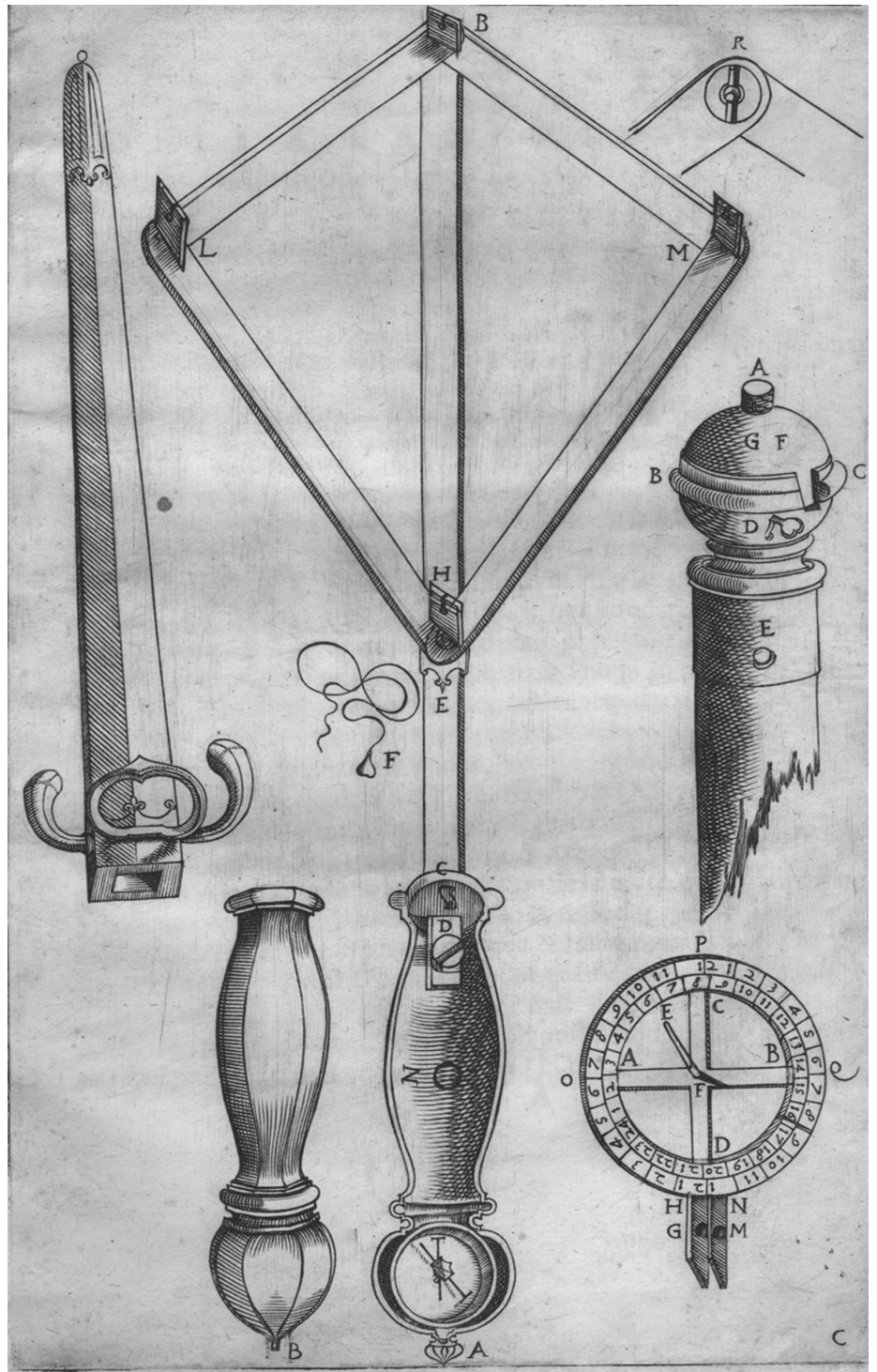

Figure 4. The radio latino illustrated in Egnatio Danti and Latino Orsini, Trattato del radio latino, Rome, 1583.

Assumptions we might naturally adopt for locating and characterizing the instrumentation of the sixteenth century thus will not always work. A further and perhaps more familiar example is the astrolabe, where the most common and seemingly most natural characterization that is frequently given presents it as a model of the heavens, a representation of the motions of the stars and the Sun in the Ptolemaic system (Figure 6). There is little evidence that this was the attitude of users in the period. However compelled we feel to see the rotation of the rete around the pole as the 


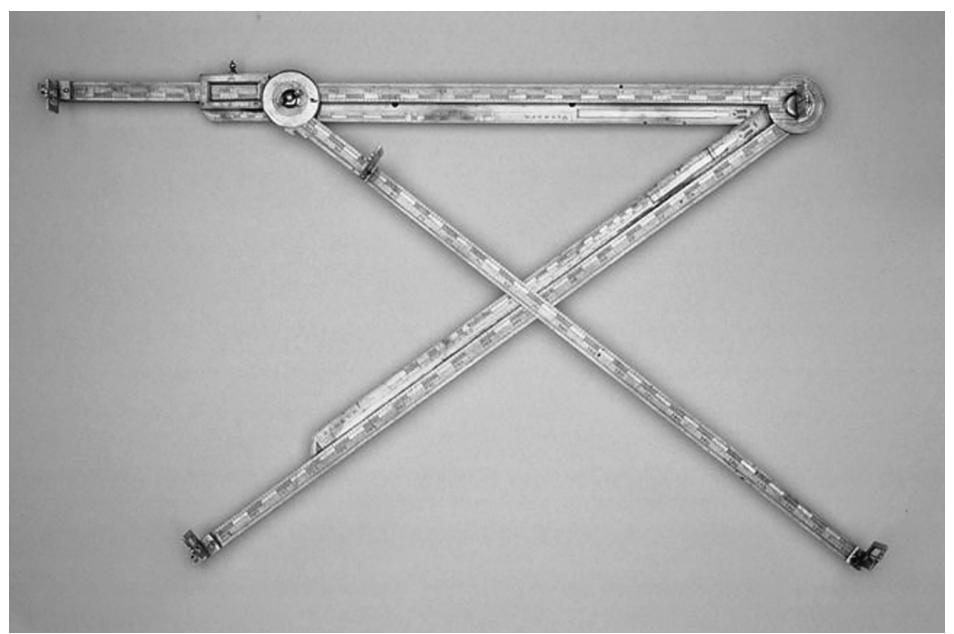

Figure 5. Triangulation instrument used, for example, for range-finding in gunnery, by Erasmus Habermel. Museum of the History of Science, inventory no. 47741.

representation of a geocentric cosmology, we do not find it in the explanatory books of the time, which concern themselves very much with doing rather than knowing. They generally follow the pioneering example of Johann Stöffler's Elucidatio fabricae ususque astrolabii of 1513, where the whole of the first section tells the reader how to construct an astrolabe and the second-dealing with its use - is a series of worked examples of how to solve certain problems and extract answers by manipulating the instrument thus constructed. ${ }^{8}$ Stöffler does not even outline what the instrument is for until he has finished his instructions on how to make it, and when he does get to a definition of the astrolabe, he says simply that it is a plane (that is, flat), round instrument, marked with many circles and lines, useful for many astronomical and geometrical operations. There is not much encouragement here for our common characterization of the astrolabe as a model.

If a model of the heavens is not in keeping with the period, what characterization might we use instead? For help with this we might look again at the Epact collection, where we will find other types of astrolabe with different projections. The traditional rete and latitude plates were replaced by a projection of the whole heavens and combinations of pivoted and sliding rules for locating points within these projections. These were 'universal' instruments, designed to work in any latitude. Leaving aside Islamic precedents, so far as European instruments are concerned, these are introductions of the sixteenth century and are due to men like Gemma Frisius and Juan de Rojas, who was associated with the mathematical school Gemma fostered at Louvain, which was also active in cartography and the application of similar projections to map-making.

8 Johann Stöffler, Elucidatio fabricae ususque astrolabii, Oppenhein, 1513, and subsequent editions. 


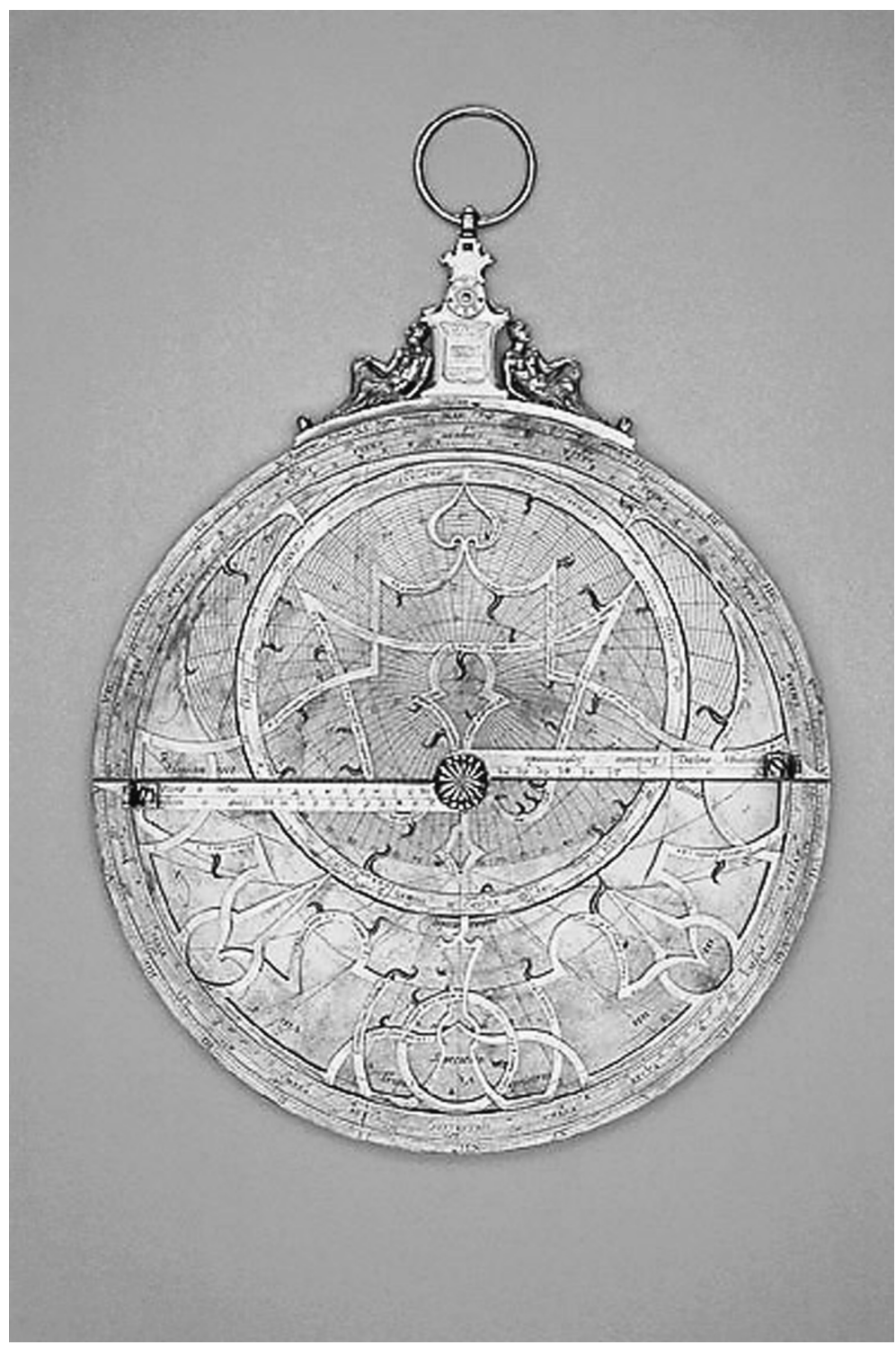

Figure 6. Astrolabe signed 'Regnerus Arsenius', made in 1565, in the Louvain workshop founded by Gemma Frisius. Museum of the History of Science, inventory no. 53558.

When projections such as these are used as the basis of an astrolabe, there is no inclination to think in terms of modelling, and of course that is correct. There is no similarly satisfying cosmological gloss that can be given to the manipulations the user performs on this astrolabe to yield answers to his astronomical or astrological problems. But it should be noted, and again there are examples in the Epact database, that such a projection may well be offered on one side of an instrument that has the rotating 


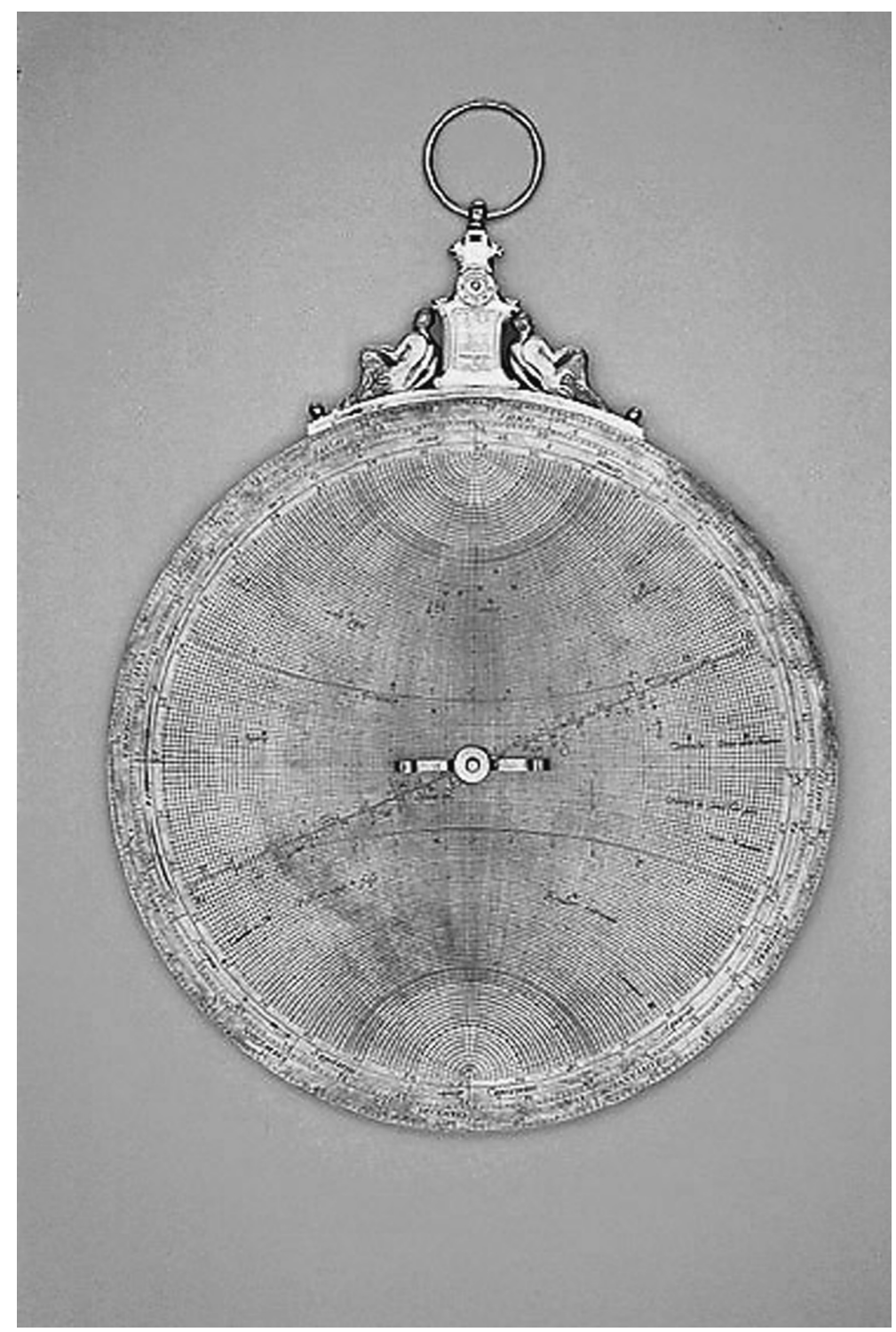

Figure 7. The universal projection on the back of the instrument illustrated in Figure 6. Museum of the History of Science, inventory no. 53558.

rete design on the other side as an alternative (Figure 7). This does not create an incoherent instrument - one combining contradictory models of the heavens - because it is not an instrument of cosmology.

This tolerance of apparent contradiction links directly with contemporary cartography, where again a range of projections are in use that coexist without incoherence because they inhabit the world of mathematical practice and not of natural philosophy. 
The alternative and coexisting astrolabe projections mirror the alternative and coexisting projections used in cartography, in the same period and by the same practitioners, to present the terrestrial world. In Gemma's book on his 'catholic astrolabe', as he calls it, one that can be used in any latitude, he discusses the different projections used to construct astrolabes, and the variety also that are employed in cartography. On the apparent liberties taken by these inconsistent geometers, he explains precisely that this is not natural philosophy: 'we can do by geometrical invention', he says, 'what is not permitted in the natural world'. ${ }^{9}$

Gemma emphasizes projection as a geometrical technique that unifies a variety of practical arts, citing its use in perspective drawing, cartography and instrumentmaking. His example is instructive for historians, for one aspect of the interconnectedness of the various mathematical arts is their range of shared techniques, both intellectual and material. The characterization we give to the products of the different mathematical arts should be consistent, not least because the books of the practitioners are forever preaching their unity and coherence, while their careers demonstrate this coherence in practice. Our characterization should work across the range.

Gemma himself offers one possibility when he refers to the astrolabe as 'typus sphaerae materialis in plano' - that is, a 'type' of the material sphere in a flat surface. ${ }^{10}$ Typus is used elsewhere in practical mathematics, for instance to refer to a map, as in the 'Typus Orbit Universalis' title to world maps by mathematicians such as Martin Waldseemüller, Peter Apian or Sebastian Münster. Other words are used to describe an astrolabe, such as 'instrumentum', 'organon' or 'analemma', depending on what aspect of its character or operation is under consideration, such as its use as an instrument for solving problems, or its projective properties. Typus might be used when what is under discussion is the relationship between this mathematical product and the material world, and while a 'type' can simply mean a figure or visual representation in a fairly straightforward way, it can suggest a more esoteric relationship with the original, in which information is encoded in a systematic manner, to be extracted by the skilled operation of the adept. This latter sense is closer to the use of projections and instruments based on projections.

A sympathetic understanding of the nature and manufacture of maps will extend the range of mathematical product that must be accommodated to our understanding of sixteenth-century instrumentation. To demonstrate the continuity between instruments and maps, we might look at the careers of the practitioners and their unquestioning and unproblematic integration of fields of work that have been separated by subsequent disciplinary practices. We could also note that the brass objects in the Epact collection are an inadequate population for representing mathematical practice, note that the engraving skills of practitioners resulted in a wider range of product, and explore the boundaries of instrument collections to see whether we can delineate a more natural population.

9 Gemma Frisius, De astrolabo catholico liber, Antwerp, 1556, f. 4v.

10 Gemma Frisius, op. cit. (9), f. 7. 
While collections will be an important resource in identifying this natural population, they must be assessed with as much critical care as we would use with any historical source, for there is much about collections that is artificial, in the sense that they may not offer us an accurate reflection of mathematical practice in the period when the instruments were made. There are many sources of distortion; we must take account of the motives and circumstances of collection-building, of the differential survival of metal instruments, the attractiveness of gilt brass to collectors and museums, the separation of the histories of cartography and science, and the separation of books in libraries, maps in map rooms and instruments in museums.

Although the great majority of the instruments in the Epact collection are made of metal, a few are in paper and these stand for the great many paper instruments from the period that have been lost. ${ }^{11}$ Many of the paper instruments that have survived have done so because they are attached to the pages of books, or more simply are pages in books. It might be said that the Epact project was remiss in not including these bookbound paper instruments in the database, as this would have been an effective way of recognizing the continuity between paper and metal instruments and removing at least one of the artificial distinctions between mathematical products. While acknowledging the legitimacy of this criticism, it can be said in mitigation that including instruments in books would have raised a major difficulty, because it is not at all clear which woodcuts or plates from books should be included and which omitted. Those with moving parts are straightforwardly instruments, but there are many other pages whose status is more debatable. Sixteenth-century books on instruments contain many fine and detailed illustrations and we should avoid taken-for-granted assumptions about what these illustrations are for. This was a new genre of text, at least in print, so we ought to ask why these illustrations are included and ignore our inherited assumption about their status.

From an early stage in the history of books about instruments, beginning with Stöffler, they contain illustrations of great detail - it might even be said of every detail (Figure 8). Every scale described in the text is reproduced in the illustration exactly as it would appear on the instrument. It may be misleading even to think of them as illustrations at all, to be constructing this gap between the impression on the page and some other object which the page illustrates. The text can be read as describing the instrument on the page just as easily and naturally as it might describe a brass example in the reader's hand. There is, in fact, a continuum of instances that links these two examples of mathematical product. There are brass instruments, wooden instruments, paper instruments, paper instruments with moving parts within books, woodcuts within books where there are no moving paper parts but where there is an anchored thread for relating scales to each other, there are woodcuts without threads but where the accompanying text invites the reader to use the instrument on the page by

11 For paper instruments, see A. J. Turner, 'Paper, print and mathematics: Philippe Danfrie and the making of mathematical instruments in late 16th century Paris' in Studies in the History of Scientific Instruments (ed. C. Blodel, F. Parot, A. Turner and M. Williams), London, 1989, 22-51; O. Gingerich, 'Astronomical paper instruments with moving parts', in Making Instruments Count (ed. R. G. W. Anderson, J. A. Bennett and W. F. Ryan), Aldershot, 1993, 63-74. 


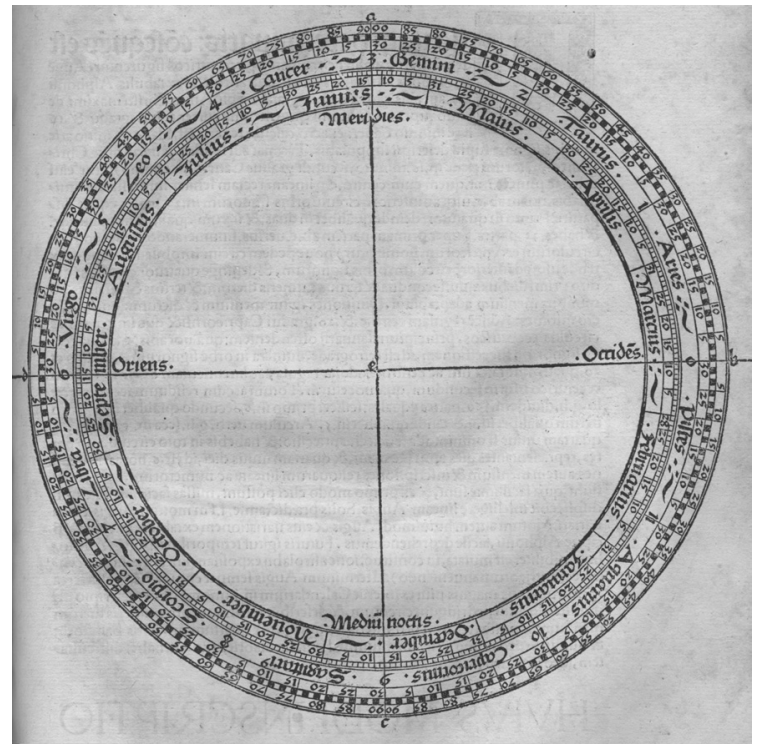

Figure 8. An example of an illustration of every detail, in this case the degree scale and zodiacal calendar of the back of an astrolabe from Johann Stöffler's Elucidatio fabricae ususque astrolabii, but typical of sixteenth-century tracts on mathematical instruments.

applying a thread or a rule to read off the scales, and there are woodcuts where there is no such instruction but which have the scales in every detail and which could certainly be used in this way. These last examples are the ones we are inclined to call 'illustrations' of something else, namely a detached and separate instrument, but to do so imputes a disjunction in the series that has no obvious justification.

There is also a strong connection between all these in terms of the craft environment for their production. A solid, dense medium is worked on with an engraving tool. If the final product is to be in brass or wood, the instrument is created directly by application of the tool; if it is to be paper, there is an intermediate stage where a print is pulled from a block of wood or a plate of copper. The designs and constructions of practical geometers, the cutting of wood-blocks and copperplates, the printing of books, of maps, of paper instruments on separate sheets, were all related activities in a world of practical mathematics that we find it difficult to integrate but whose relationship in the sixteenth century is evident in the careers of many mathematicians.

There is nothing obscure or special about the inclusion of a paper instrument in a sixteenth-century book; some of the most common astronomical and cosmographical books have instruments with moving parts, such as the many editions of Sacrobosco's Sphere and Peter Apian's Cosmographia with its many editions prepared by Gemma Frisius. There are also rare and expensive books with instruments, famously Apian's Astronomicum Caesareum. ${ }^{12}$

12 O. Gingerich, 'Apian's Astronomicum Caesareum and its Leipzig facsimile', Journal for the History of Astronomy (1971), 2, 168-77. 
This coherence across the range of mathematical product means that the way we characterize mathematical instruments should have a broader reference, and the way other aspects of sixteenth-century mathematical practice are sensitively characterized should be helpful with regard to instruments. Instrument historians must not see themselves as inhabiting a separate historical discipline, and they have things to learn as well as to teach. Historians of astronomy, for example, are familiar with the idea of a theoric, or theorica in Latin, as a geometrical construction yielding the positions of a particular planet. But we can extend this idea. A geometrical theoric of widespread applicability, used to encapsulate a relationship between appearances, from which information could be extracted by the use of certain protocols, is an idea of general relevance to mathematical practice, and could characterize not only planetary constructions but also maps and instruments.

A theoric was intended to contain and generalize measurements across space or across time, often employed mathematical techniques close to those involved in instrumentation, and could take an instrumental form. The word theorica could, for example, be used to refer to an equatorium, an instrument used to derive planetary positions by the manipulation of discs and rules. Maps to scale, introduced in the sixteenth century, were encapsulations or epitomes of information with epistemic characteristics similar to those of theorics in astronomy, as were many instruments, particularly those incorporating projections, such as astrolabes, horary quadrants and a variety of sundials. As a further example of such connections, when William Borough writes in 1581 about the possibility of reducing the differences in magnetic variation in different places to some kind of rule, he talks in terms of saving the appearances, an ambition we more readily identify with geometrical astronomy. ${ }^{13}$

The way the geometers worked across the range of applications, and employed similar mathematical techniques in each of them, ensured a coherent attitude to the status and operation of the theoric in relation to nature. As a single example, we could take Johann Werner. Though better known in the history of cartography, he is perhaps best known to historians of science on account of the 'letter against Werner' written by Copernicus. ${ }^{14}$ Werner was, like so many mathematicians of the sixteenth century, concerned both with astronomy and cartography: his translation of Book 1 of Ptolemy's Geographia, published in Nuremberg in 1514, introduced four new projections. Werner was thus familiar with the use and status of the geometrical theoric in astronomy and cartography. It is interesting that Copernicus chastises him for not respecting the authority of the ancients, Ptolemy in particular. ${ }^{15}$ That was not a problem for a cosmographer: while astronomy was still recovering the text of Almagest, first published in 1515, and assimilating the technicalities of Ptolemaic practice in astronomy, in cartography the geometers had already moved beyond Geographia, driven not least by its evident inability to deal with the voyages of discovery.

As part of the development of cartography beyond the limits of Geographia, it was Werner who suggested the cordiform or heart-shaped projection that refined and

13 W. Borough, A Discours of the Variation of the Cumpas, or Magneticall Needle, London, 1581, preface.

14 E. Rosen (ed.) Three Copernican Treatises, New York, 1959.

15 Rosen, op. cit. (14), 99-100. 
extended one of Ptolemy's projections to include the whole globe, and it was Apian who took this up and realized its full potential. ${ }^{16}$ Werner and Apian are two prominent examples, but it is not difficult to think of men fully conversant with the theorics of astronomy while being active across the range of practical mathematics. Much closer to the circle of Copernicus would be Rheticus, who was concerned with surveying, cartography and instrumentation. Johann Schöner, a teacher of Rheticus and to whom Rheticus addressed the Narratio Prima, ${ }^{17}$ was a leading astronomer who was also involved with cosmography and globe-making. Examples are easily multiplied because astronomy was part of a wider domain of mathematical science, but we have yet to take full advantage of that in the history either of astronomy or of practical mathematics.

We can say that sixteenth-century instruments are both for doing and for knowing, but only if we are careful about the kind of knowing involved. Doing tends to predominate in the presentations given in instruction manuals about the instruments, and is more evident than knowing in the designs of many of the instruments themselves. Where knowing makes a more obvious appearance, it is not the knowing characteristic of natural philosophy. The vehicle that encapsulates the relationship between mathematics and the material world, and the work that practical mathematicians do there, is characteristically the theoric. It does not deliver insight into the nature of things, but the skilled operator can apply to it a set of protocols that are part of the doing of mathematical practice and it will yield answers to problems after the manner explained, for example, in the second section of Stöfler's Elucidatio.

There is at least one point of interest for historians of science that emerges from this consideration of instruments: it expands the relevance of work done on the longestablished historical question of the cosmological status of geometrical planetary theory in the sixteenth century. A traditional area of historical curiosity encompassed what was referred to as instrumentalist and realist views of astronomical theory, Andreas Osiander's preface to De Revolutionibus, Kepler's insistence on the realist status of his planetary account and the need to support it through a physical explanation in the Astronomia Nova, the bafflement of his teacher Michael Maestlin as a true mathematician at this errant attitude of his pupil, and the general issue of the evolving role of mathematics in relation to natural philosophy. We can now suggest that this set of issues is not confined to astronomy, but instead is characteristic of the range of mathematical sciences, and could be retold in a number of areas. This gives the familiar astronomy/cosmology story a much wider relevance. It might even be suggested that the prominence historians have given to that particular strand in the more general narrative does not accord well with the priorities of the period. I have already argued the enormously greater currency of Ptolemy's Geographia in the sixteenth century compared with Almagest. ${ }^{18}$

In conclusion, I want to use this excursion into the realm of instrumentation to illuminate an old problem in the history of science - to move, in other words, from the

16 L. Bagrow, History of Cartography (ed. R. A. Skelton), London, 1960, 130.

17 Rosen, op. cit. (14), 9, 109.

18 J. A. Bennett, 'The challenge of practical mathematics', in Science, Culture and Popular Belief in Renaissance Europe (ed. S. Pumfrey, P. L. Rossi and M. Slawinski), Manchester, 1991, 176-90. 
curatorial to the historical mode of work, bringing with us what the instruments may have shown us about mathematical practice. Sensitivity to the more widespread use of something very like geometrical theorics beyond astronomy may be of use in dealing with a very particular question concerning the cosmology of William Gilbert. Gilbert gives us an interesting example of a theoric outside astronomy, in an area of importance to the emergence of the experimental natural philosophy of the seventeenth century, so this may be an instance where curatorial work can offer a contribution to what has been seen as mainstream history of science.

Gilbert was a puzzling anomaly for, for example, Alexandre Koyré, because his cosmology seems at once radically bold and disappointingly timid. He denies the existence of the celestial sphere, along with all the planetary spheres denied by Tycho, and postulates an infinite starry heaven. But, while he accepts the daily rotation of the Earth, he does not clearly embrace the annual motion. Gilbert does not deny the existence of the annual motion, he just ignores this part of the Copernican system, accepting, as Koyre puts it, only 'the least important part' of Copernicanism. ${ }^{19}$

Koyré's focus and that of later commentators, on Book 6 of De Magnete - that is, on the cosmological part - has been unfortunate. It is helpful to begin a detailed reading earlier, so as at least to take in Book 5 , on the magnetic inclination or dip. Gilbert seems to signal the importance of dip in introducing Book 5 with a particular flourish:

We come at last to that fine experiment, that wonderful movement of magnetic bodies as they dip beneath the horizon in virtue of their natural verticity; after we have mastered this, the wondrous combination, harmony, and concordant interaction of the earth and the loadstone (or magnetized iron), being made manifest by our theory, stand revealed. ${ }^{20}$

It is in Book 4, on magnetic variation, and Book 5, on inclination, that Gilbert seems closest to the practical mathematical tradition. It is here that the reader encounters the measuring instruments he used, and it is here that he seems most familiar with the empirical and instrumental tradition already well established among the English mathematical practitioners in their engagement with navigational magnetism. It has even been argued that some of the more mathematically proficient chapters in these books were actually written by Gilbert's friend, the mathematician Edward Wright. ${ }^{21}$

One thing to realize is that although variation and dip are often seen as complementary deviations from a magnetic norm, they have no such parallel status for Gilbert. Dip had a much more profound role in his natural philosophy. Variation is a mere deviation contingent on local irregularities. Dip, on the other hand, is a fundamental characteristic of magnetic bodies, as fundamental as direction, alignment with the poles, that is. Just as there is variation from the true direction, so there is variation from the true dip, again due to local irregularity.

Gilbert classifies magnetic characteristics as motions: direct motions such as attraction, or more properly speaking coition, or rotary or turning motions such as direction

19 A. Koyré, From the Closed World to the Infinite Universe, Baltimore, 1968, 55-6.

20 W. Gilbert, De Magnete (tr. P. Fleury Mottelay), New York, 1958, 275.

21 S. P. Pumfrey, 'William Gilbert's magnetic philosophy, 1580-1684: the creation and dissolution of a discipline', Ph.D. dissertation, Warburg Institute, University of London, 1987. 


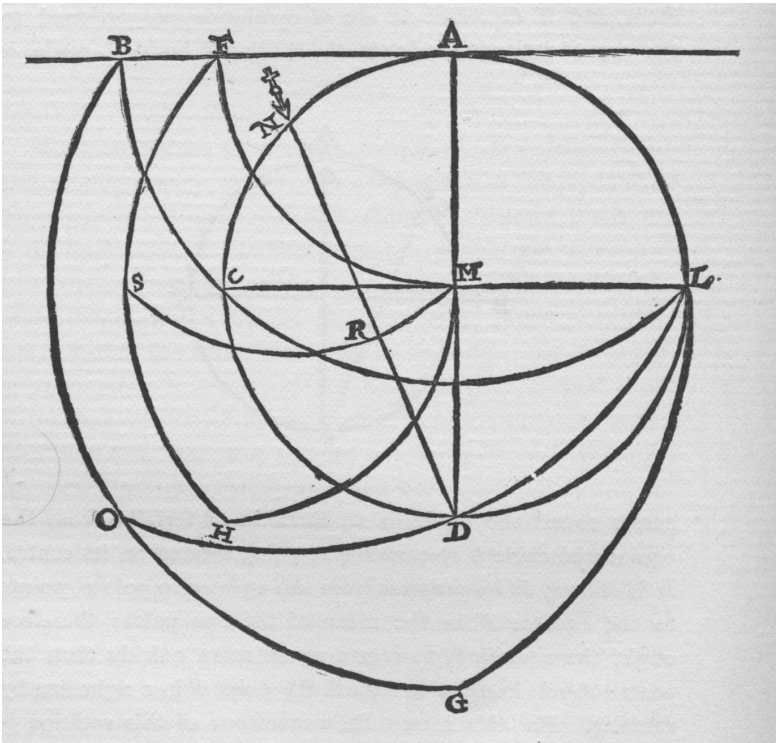

Figure 9. The theoric of dip: Gilbert's construction in De Magnete for the dip at latitude forty-five degrees.

or dip. In Book 5 he offers a global account of dip, and it is here that we find a theoric, which relates dip to latitude. While not fully clarified by the accompanying text, it is a geometrical pattern that yields the dip everywhere from the equator to the pole, and so by symmetry, everywhere on the globe. Gilbert (or Wright) does not give a very developed physical account from which such a pattern is derived. It has three fixed points: at the equator the dip is zero and at the poles it is ninety degrees and, according to Gilbert, at a latitude of forty-five degrees the needle points to the distant extremity of the equatorial diameter (Figure 9). Again no particular physical mechanism is offered for this intermediate point, beyond the comment that when the needle is midway in its progress from equator to pole, during which it turns through 180 degrees or twice the angle it travels with respect to the centre, it must respect the mean position between the poles. We are not told why this mean position is at the Earth's circumference, instead of some other point in the equatorial plane; perhaps Gilbert or Wright had a number of reported measurements that the theoric had to match as well as possible. The construction he devises is applied to all degrees of latitude in a large folded plate in the manner of contemporary mathematical instruments (Figure 10).

Let us admit this theoric and move on, for what happens after this is particularly interesting. Indeed Gilbert signals that we are about to hear something really exciting:

having with divers and manifold contrivances laboured long and hard to get at the cause of this dip, we have by good fortune discovered a new and admirable science of the spheres themselves - a science surpassing the marvels of all the virtues magnetical. ${ }^{22}$

22 Gilbert, op. cit. (20), 304. 


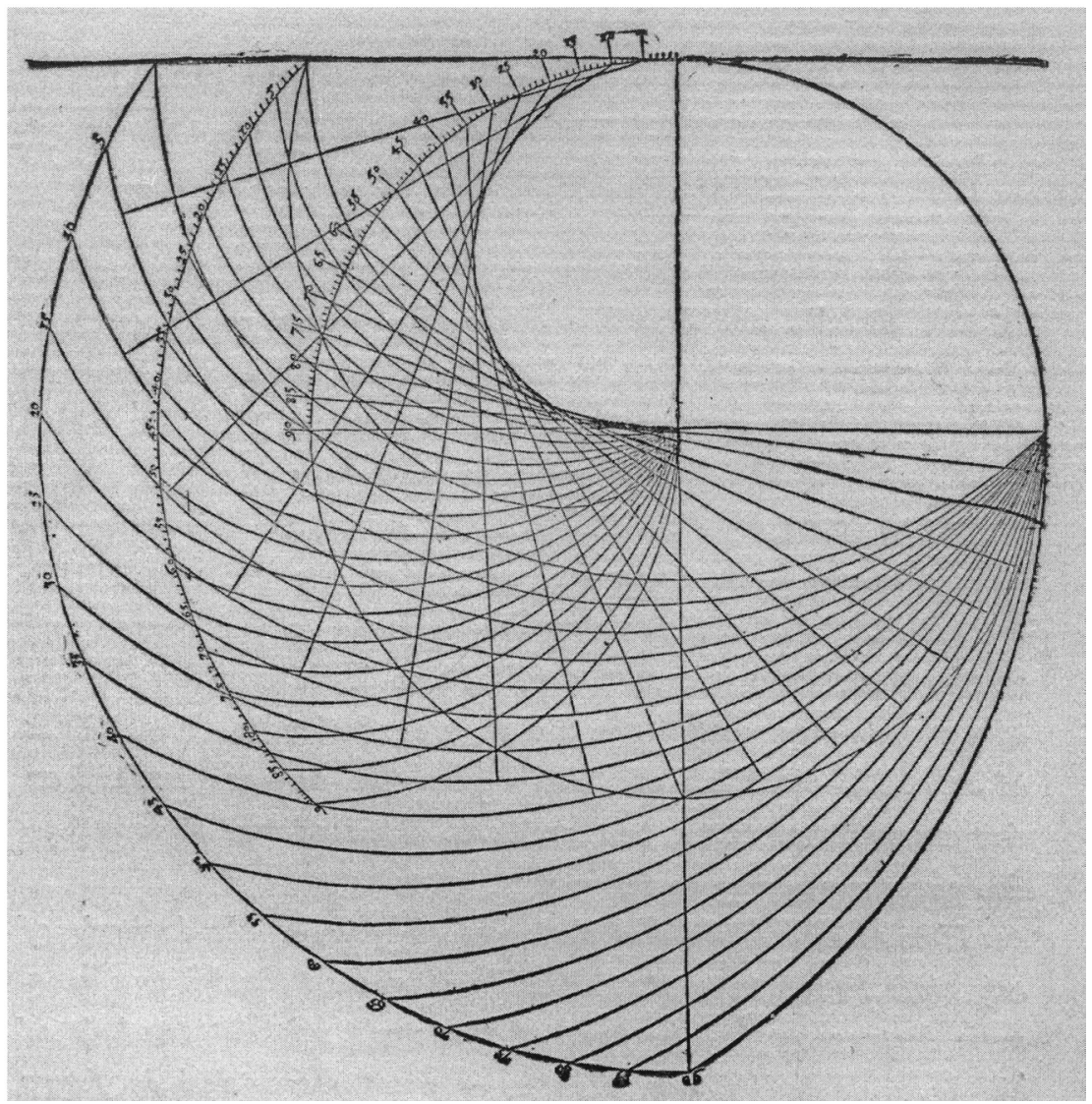

Figure 10. Gilbert's theoric of dip applied to every degree of latitude.

What can he mean? What are these spheres? Gilbert is supposed to be celebrated for removing even the last of the spheres, so how can he be reintroducing them here?

This new science is a science of the spheres because the form of a magnetic sphere extends beyond its corporeal boundary; its magnetic action - that is, its incitement to magnetic motion - acts beyond its surface in an infinite set of 'effused spheres'. Around a spherical magnet, we might imagine concentric spheres - formal spheres, not corporeal ones. He draws three in his explanatory diagram, but this is only for illustration: as Gilbert says, 'they may be imagined as infinite' (Figure 11). ${ }^{23}$ At this point we hear the authentic voice of Gilbert, as the discussion moves very clearly and self-consciously from a geometrical theoric to a natural philosophy dealing with causes: "the mind that has diligently studied this natural philosophy will discover the definite causes of the movements and revolutions'. ${ }^{24}$ Talk of movements and revolutions is unexpected here, because this is still Book 5, dealing with spherical lodestones, not with the magnetic

23 Gilbert, op. cit. (20), 305.

24 Gilbert, op. cit. (20), 304. 


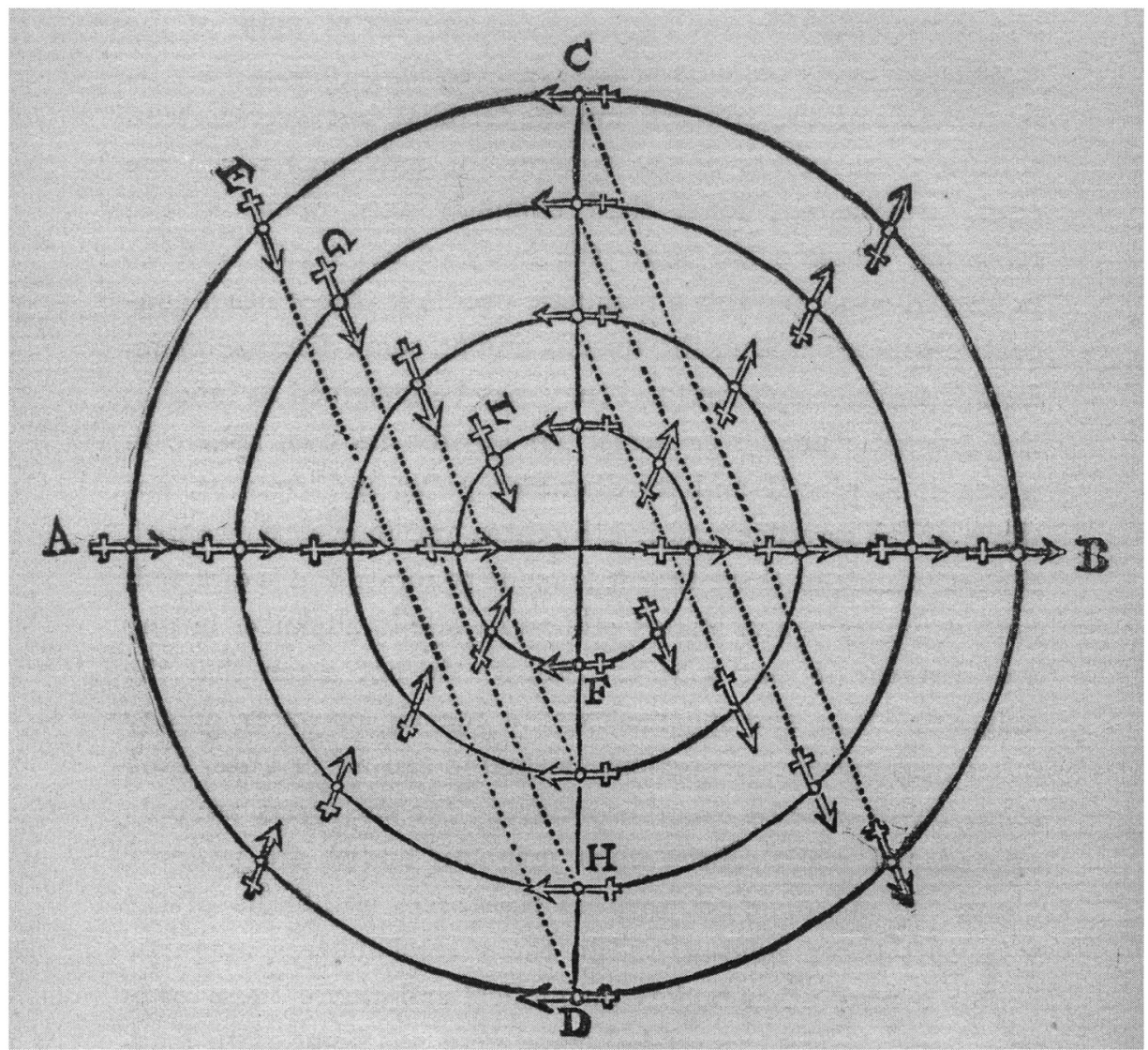

Figure 11. Three of the infinite number of 'effused spheres' around a spherical lodestone, as illustrated in Gilbert's De Magnete.

Earth, but such talk does seem to point to a cosmic relevance for the argument. These will become, in the old sense, motive spheres, but they are not material and they do not act mechanically. Their action is like that of a soul.

Remember that for Gilbert the magnetic movement of dip is a species of revolution. He applies his dip theoric to each of these spheres, each needle orientating with respect not to the corporeal lodestone but to the formal sphere in which it is placed. Dip is not a result of attraction, but of the power of the lodestone to effect a revolution in a magnetic body. Here he proves this by suspending a needle attached to a cork in water, not on the surface but within the liquid. It rotates to its appropriate alignment, but it is not drawn towards the Earth.

Gilbert is now able to talk of revolutions at the surfaces of these immaterial spheres: 'so all the revolutions in the spheres to the termini of the spheres are such as are the revolutions at the surface of the terella or to its termini'. As though to push the cosmological allusions a little further, he points out that as a magnetic needle is carried 
around a terella, following one of these 'effused spheres', the needle makes two complete revolutions for one circuit of its centre, 'like an epicycle round its circle'. ${ }^{25}$

Now we are ready for the final, cosmological chapter of De Magnete. The ingredients are well known, since the cosmology is in the commonly read Book 6. Gilbert mentions crystalline spheres, though of course now only to dismiss them in material terms.

The Earth is a great magnet, and it rotates by a magnetic movement, a rotation incited by a magnetic soul. The ancients had traditionally ascribed souls to the celestial spheres; now Gilbert believes he can make sense of this venerable notion. For Gilbert to say that a body is ensouled is to say that it acts immaterially by a formal presence beyond its corporeal limits. This is not a magical animism in what he would think of as the vulgar sense, but an account of action beyond a body's corporeal limits by postulating a formal presence that affects cognate bodies. There are no material spheres in such a world, and nothing even to confine the celestial region, so the heavens are infinite. The daily rotation of the celestial sphere was incredible, but the rotation of the infinite heavens impossible and absurd. Rather it is the Earth that rotates, and this rotation is a magnetic movement - the rotation of a magnet on the formal effused sphere of a magnetic globe.

As far as the Earth's rotation was concerned, this magnetic globe was the Sun: the Earth rotates 'because of the effused spheres of the solar influence', she 'revolves in a circle towards the sun ${ }^{{ }^{26}}{ }^{6}$ Whether or not there is an annual component, the Earth's motion belongs with and is part of the general planetary motion through the action of the Sun's effused spheres:

The sun (chief inciter of action in nature), as he causes the planets to advance in their courses, so, too, doth bring about this revolution of the globe [i.e. the rotation of the Earth] by sending forth the energy of his spheres - his light being effused. ${ }^{27}$

With specific reference to the different motions of revolution and rotation, Gilbert says, 'Thus each of the moving globes has circular motion, either in a great circular orbit or on its own axis or in both ways. ${ }^{, 28}$ It is difficult to imagine which of the planets was thought to have both motions if not the Earth. Indeed elsewhere in Book 6 Gilbert specifically says he intends to concentrate on the diurnal rotation and to 'pass by' the Earth's other movements. ${ }^{29}$

Recent historians have, in fact, come to the general opinion that Gilbert held to the annual revolution as well as to the diurnal rotation of the Earth. In the posthumously published De Mundo, again he is not explicit, but the cosmology he describes once more prioritizes the Sun as the principal source of motion, acting through its effused spheres and thus moving the Earth as well as the other planets. Since it is the formal spheres concentric with the Sun that move the Earth, his scheme is most compatible with the Earth in motion around the Sun, but in the diagram of the universe in the

25 Gilbert, op. cit. (20), 306-7.

26 Gilbert, op. cit. (20), 330, 333.

27 Gilbert, op. cit. (20), 333.

28 Gilbert, op. cit. (20), 334.

29 Gilbert, op. cit. (20), 327. 


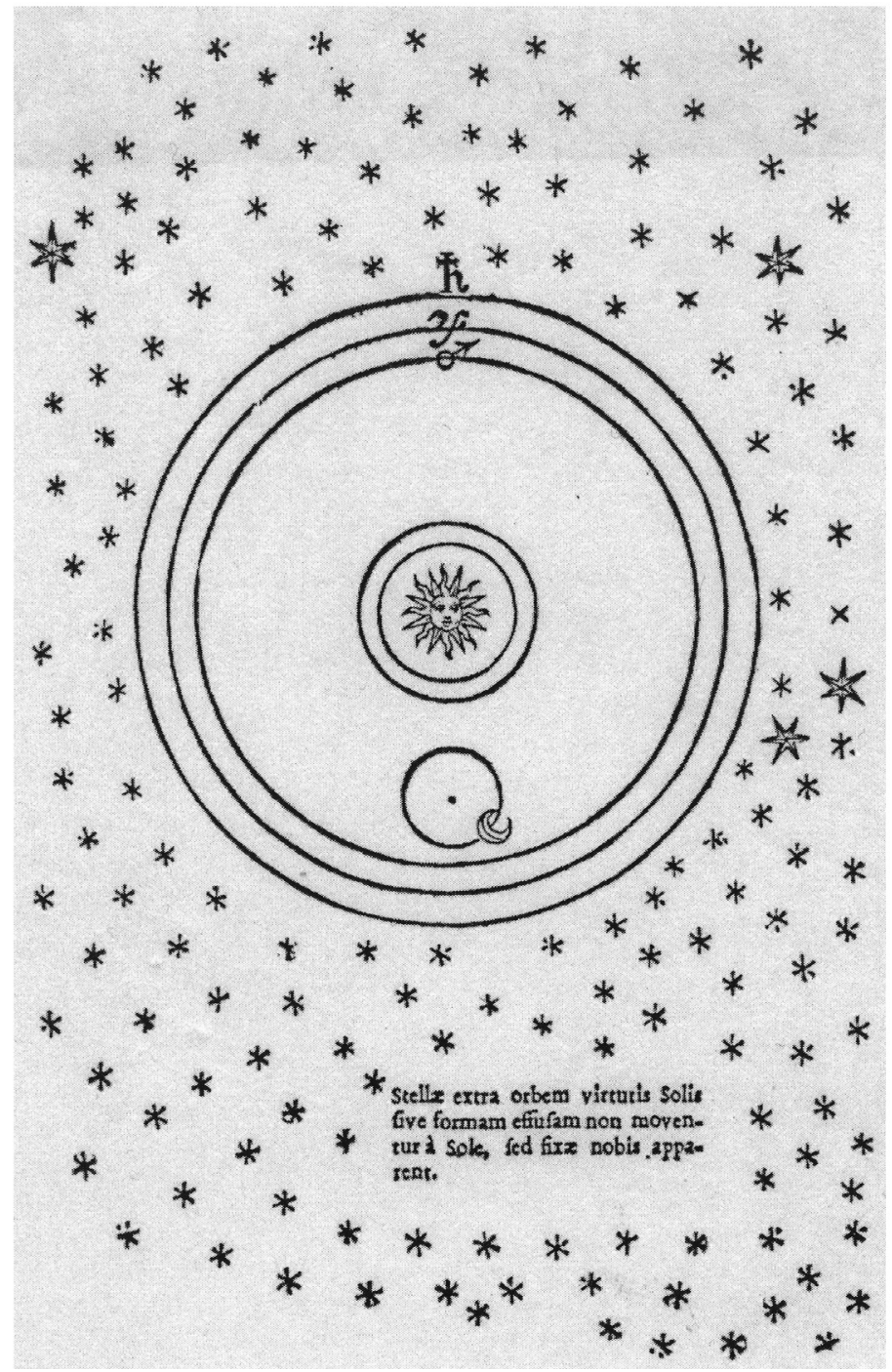

Figure 12. The diagram of the cosmos in Gilbert's De Mundo.

De Mundo, a planetary path is indicated for neither the Earth nor the Sun, so technically ambiguity is preserved (Figure 12). Freudenthal has noted, however, that if it is the Sun that moves around the Earth, the planetary paths would enter the region of the stars, and stellar parallax would be evident. ${ }^{30}$

More importantly, we now know that the circles on this diagram do not represent planetary paths so much as effused spheres, including the small sphere around the Earth

30 G. Freudenthal, 'Theory of matter and cosmology in William Gilbert's De magnete', Isis (1983), 74, $22-37$. 
and intersecting the Moon. It is not clear why the sphere on which the Earth is positioned has been omitted, but whatever the reason, if the circle linking the Sun and the Earth were to be drawn-since it represents a sphere, rather than a planetary path - it could only be centred on the Sun and pass through the Earth: Gilbert's text makes it absolutely clear that it is the formal sphere of the Sun that moves the Earth, not the other way round. Gilbert's caption, included among the stars, confirms that the circles stand for the effused spheres rather than the usual planetary paths: 'The stars beyond the sphere of virtue of the Sun or its effused form are not moved by the Sun, but appear to us fixed. ${ }^{31}$

Now it may be that the identification of these circles with the effused spheres, rather than with the conventional planetary theorics, and the more complete understanding of Gilbert's cosmology was there to be read without having armed ourselves with a greater familiarity with the techniques of the mathematical sciences. But it was a more careful reading of the mathematical agenda and a characterization of contemporary mathematical products, including instruments, that helped to identify the proper location of the mathematical theoric - not in this diagram from De Mundo but in Book 5 of De Magnete where Gilbert introduced the theoric of dip. We were then able to see where he shifts his attention to natural philosophy, a shift that made this a diagram of effused spheres and not of planetary circles, as it has been assumed to be. Admitting a wider range of mathematical arts and sciences from the sixteenth century into a shared field of study will both reflect the realities of the period and create a greater space for collaborative work between historians and curators. In De Magnete we have seen the crucial move from mathematical theoric to natural philosophy in the case of navigational magnetism. This observation both enriches and is enriched by our greater familiarity with the equivalent move in astronomy almost a decade later, the publication of Kepler's Astronomia Nova.

31 W.Gilbert, De mundo nostro sublunari philosophia nova, Amsterdam, 1651; S. Kelly, The De Mundo of William Gilbert, Amsterdam, 1965. 